\title{
Sec13 induces genomic instability in U20S cells
}

\author{
Choong-Ryoul Sihn ${ }^{1}$, Eun-Jung Suh ${ }^{1}$, \\ Kee-Ho Lee ${ }^{2}$ and Sang Hoon Kim ${ }^{1,3}$ \\ ${ }^{1}$ Department of Biology \\ College of Sciences, Kyung Hee University \\ Seoul 130-701, Korea \\ ${ }^{2}$ Laboratory of Molecular Oncology \\ Korea Cancer Center Hospital \\ Seoul 139-240, Korea \\ ${ }^{3}$ Corresponding author: Tel, 82-2-961-9208; \\ Fax, 82-2-964-1079; E-mail, shkim@khu.ac.kr
}

Accepted 9 June 2005

Abbreviations: APC, anaphase promoting complex; COP II, coat protein II; ER, endoplasmic reticulum; GFP, green fluorescence protein; NPC, nuclear pore complex

\begin{abstract}
Sec13p has been known as an endoplasmic reticulum-Golgi transport protein. Recently, it has also been shown to be required for the formation of septation in the fission yeast Schizosaccharomyces pombe. In the present study, we focused on the role of a human homolog of Saccharomyces cerevisiae SEC13, Sec13 protein during mitosis in U2OS cells. We found that the expression of Sec13 was constant throughout the cell cycle, and localized to the kinetochores at metaphase during mitosis. By using green fluorescent protein technology, we observed that Sec13 is required for evasion of mitotic arrest in response to spindle damage, leading to G1-like phase and apoptotic cell death. In addition, cells expressing exogenous Sec13 showed giant nuclei compared to endogenous ones in the absence of nocodazole. These results demonstrate that Sec13 is involved in the regulation of the metaphase/anaphase transition and may be functionally associated with mitotic machinery to maintain genomic stability during mitosis.
\end{abstract}

Keywords: Sec13; mitosis; genomic instability; U2OS cells; nuclear pore complex

\section{Introduction}

Yeast Sec13p is a component of the yeast coat protein II (COP II) complex mediating the process of vesicle budding from endoplasmic reticulum (ER) to Golgi apparatus (Pryer et al., 1993; Lederkremer et al., 2001). The mammalian homolog of yeast SEC13 is isolated and mapped at a chromosome 3p24-p25 physically near the tumor suppressor protein Von Hippel-Lindau locus (Swaroop et al., 1994). Like yeast Sec13p, human Sec13 protein is essential for the transport of ER to Golgi and is a component of mammalian COP II complex as a coat and adapter protein (Tang et al., 1997; Lederkremer et al., 2001). In addition, the subcellular localization of Sec13 has been observed on the membranes of the nuclear pore in yeast and mammalian cells (Siniossoglou et al., 1996).

Recently, there are reports showing that Sec13 associates with Nup107, Nup160, Nup133, and Nup96 as a nuclear pore complex (NPC) (Boehmer et al., 2003; Enninga et al., 2003). When Nup358, one of the NPC complex components, is knocked-down for its expression, the structure and function of kinetochores becomes abnormal, leading to chromosome missegregation (Salina et al., 2003). In addition, Nup107 also localizes at kinetochores during mitosis (Belgarch et al., 2001). Interestingly, in yeast, Mad1 and Mad2, components of the spindle checkpoint, localize on the nuclear membrane during interphase and bind to the NPC complex (Campbell et al., 2001; louk et al., 2002).

The spindle checkpoint is like a surveillance system to control chromatid segregation during mitosis. However, there is no data to explain the role of the NPC complex during mitosis in mammalian cells. There is only a report that in fission yeast, the Sec13 mutant sensitive to dimethylsulphoxide (DMSO) fails to cleave the division septum, an event at the end of the cell cycle (Poloni and Simanis, 2002). Therefore, in the present study, we focused on whether Sec13 is involved in regulating mitotic progression in mammalian cells.

\section{Materials and Methods}

\section{Cell culture and synchronization}

Human osteosarcoma U2OS cells were cultured in Dubelcco's modified essential medium (DMEM) supplemented with $10 \%$ fetal bovine serum, antibiotics and incubated in humidified incubator at $37^{\circ} \mathrm{C}$ with $5 \%$ $\mathrm{CO}_{2}$. For synchronization, cells were arrested at $\mathrm{mi}-$ tosis by treatment of nocodazole and then harvested detached cells by mechanically shaking off. Synchronized cells were released in drug-free fresh medium and collected at the indicated time points. 


\section{Transient transfections and mitotic index}

The pEGFP-Sec13 construct was made by subcloning a 969 base pair of Sec13 cDNA EcoRI/BamHI fragment into pEGFP. Transfections into U2OS cells were performed using Lipofectamine transfection reagent (GIBCO-BRL) according to manufacturer's protocol. U2OS cells $\left(1 \times 10^{5}\right)$ were incubated for $3 \mathrm{~h}$ with Lipofectamine reagent-DNA complexes, with the optimal ratio determined to be $30 \mu$ of Lipofectamine reagent/ $3 \mu \mathrm{g}$ of DNA. For determination of GFP fluorescence signal, cells were harvested $24 \mathrm{~h}$ after transfection and were observed by fluorescence microscope. To determine the mitotic index, cells were treated with the $100 \mathrm{ng} / \mathrm{ml}$ dose of nocodazole for 16 $\mathrm{h}$ to increase the numbers of mitotic cells. After fixing with $4 \%$ paraformaldehyde, cells were stained with DAPI for nuclear staining. Mitotic nuclei were readily apparent through microscopic analysis of the stained nuclei.

\section{Western blot analysis}

The cell extracts were subjected to electrophoresis in $12 \%$ gradient SDS-PAGE gels. The proteins were transferred to a polyvinylidene difluoride membrane and blocked in $5 \%$ milk for $1 \mathrm{~h}$ followed by incubation with anti-Sec13 antibody at $4^{\circ} \mathrm{C}$. A horseradish peroxidase-conjugated secondary antibody (Santa Cruz Biotechnology) was applied for $20 \mathrm{~min}$. The membranes were washed in $1 \times$ Tris-buffered saline with $0.1 \%$ Tween 20 (Sigma) and visualized by enhanced chemiluminescence (ECL; Amersham Biosciences). Sec13 antibody was generously provided by $\mathrm{Dr}$. Bor Luen Tang (Institute of Molecular and Cell Biology, Singapore).

\section{Flow cytometry analysis}

U2OS cells were plated onto $10-\mathrm{cm}$ dishes $\left(1 \times 10^{6}\right.$ cells/dish) in DMEM containing $10 \%$ fetal bovine serum and were treated with microtubule disrupting agents. At $16 \mathrm{~h}$, cells were harvested and fixed with $70 \%$ ethanol. After centrifugation, cells were resuspended in PBS containing $2 \mathrm{mg} / \mathrm{ml}$ RNase (Sigma) and stained with $0.1 \mathrm{mg} / \mathrm{ml}$ propidium iodide (Sigma). For each sample, 10,000-20,000 GFP-positive cells were analyzed for DNA contents and cell cycle phase distribution using a FACSCalibur instrument (Becton Dickinson). The data were analyzed using CELLQuest (Becton Dickinson).

\section{Immunofluorescence assay}

U2OS cells expressing GFP-Sec13 fusion protein were cultured on coverslips and fixed with $4 \%$ paraformaldehyde for $15 \mathrm{~min}$, and washed three times with PBS. After blocking with 10\% horse serum, primary antibodies against $\alpha$-Sec13 and $\alpha$-Crest were added in humidified chamber for $1 \mathrm{~h}$. After washing three times with PBS containing $0.1 \%$ Triton X-100 (PBS-T), secondary antibodies conjugated to FITC or
TRITC (Sigma) were added at a dilution of $1: 100$ in PBS-T and incubated for $45 \mathrm{~min}$. DAPI $(0.1 \mathrm{ug} / \mathrm{ml})$ was used to detect nuclei. Samples were washed three times with PBS-T, mounted and visualized on a Carl Zeiss LSM510.

\section{Results and Discussion}

\section{The expression of Sec13 during the cell cycle}

To investigate the expression of human $\operatorname{Sec} 13$ in a cell cycle-dependent manner, U2OS cells arrested at metaphase were released and harvested at different time points. As shown in Figure $1 \mathrm{~A}$, the protein level of Sec13 appeared to be steady during the cell cycle. Since ER or Golgi-related proteins are expressed constantly during the cell cycle (Asada et al., 1995), the expression of Sec13 is an analogous pattern of the typical Golgi-related protein. Since in a previous study yeast SEC13 has been reported for its essential role in the septum formation (Poloni and Simanis, 2002), we investigated the localization of endogenous Sec13 in U2OS cells with Crest antibody as a kinetochore marker. As shown in Figure $1 \mathrm{~B}$, during interphase the intensity of Sec13 was detected in the nucleus as well as in the ER and Golgi complex consistent with previous studies (Tang et al., 1997; Zaal et al., 1999), whereas the signal of Crest antibody was detected in an intense speckled nuclear staining. One of the Golgi marker proteins, galactosyltransferase, also shows a similar pattern like Sec13. Golgi fragment fluorescence distributed diffusely during the cell cycle (Zaal et al., 1999). The diffused staining pattern is a general phenotype for Golgi membrane proteins during the cell cycle. When cells entered mitosis, Sec13 remained at nucleoplasm, which was a similar pattern to that of Crest. At metaphase, the intensity of Sec13 which could be detected at kinetochore coincided with a kinetochore marker. The signal of Sec13 kept toward telophase and occupied the spindle apparatus around segregated sister chromatids including the midzone of the spindle. At telophase, intense fluorescent staining was also observed in the region of the intracellular bridge. These observations are consistent with that Sec13 yeast mutants fail to cleave the division septum (Poloni and Simanis, 2002), suggesting that human Sec13 could be required for mitotic progression in mammalian cells.

\section{Mitotic progression induced by Sec13}

Interestingly, during mitosis the localization pattern of Sec13 is similar to mitotic checkpoint proteins such as Mad2 (Li and Benezra, 1996). These mitotic proteins are associated with the chromosomes unattached to the microtubules. We, therefore, investigated to determine the role of the Sec13 on the spindle checkpoint activation. U2OS cells transiently expressing GFP-Sec13 fusion protein were treated with the microtubule-disrupting drug, nocodazole (100 
A

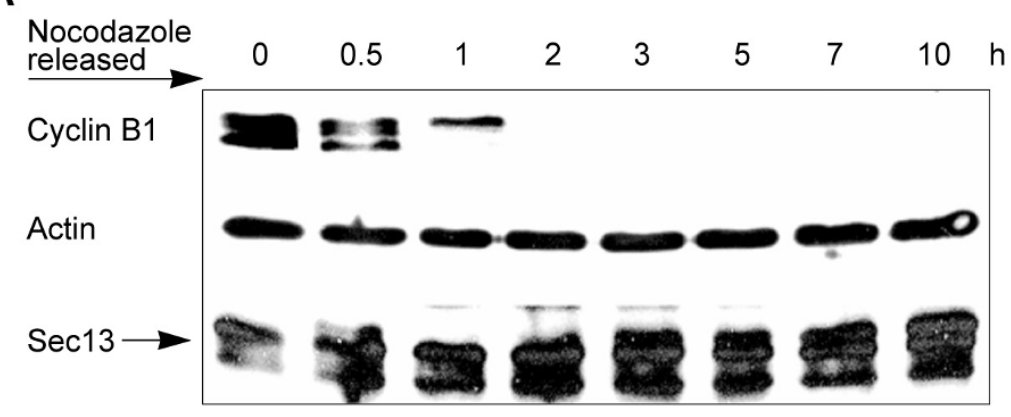

B

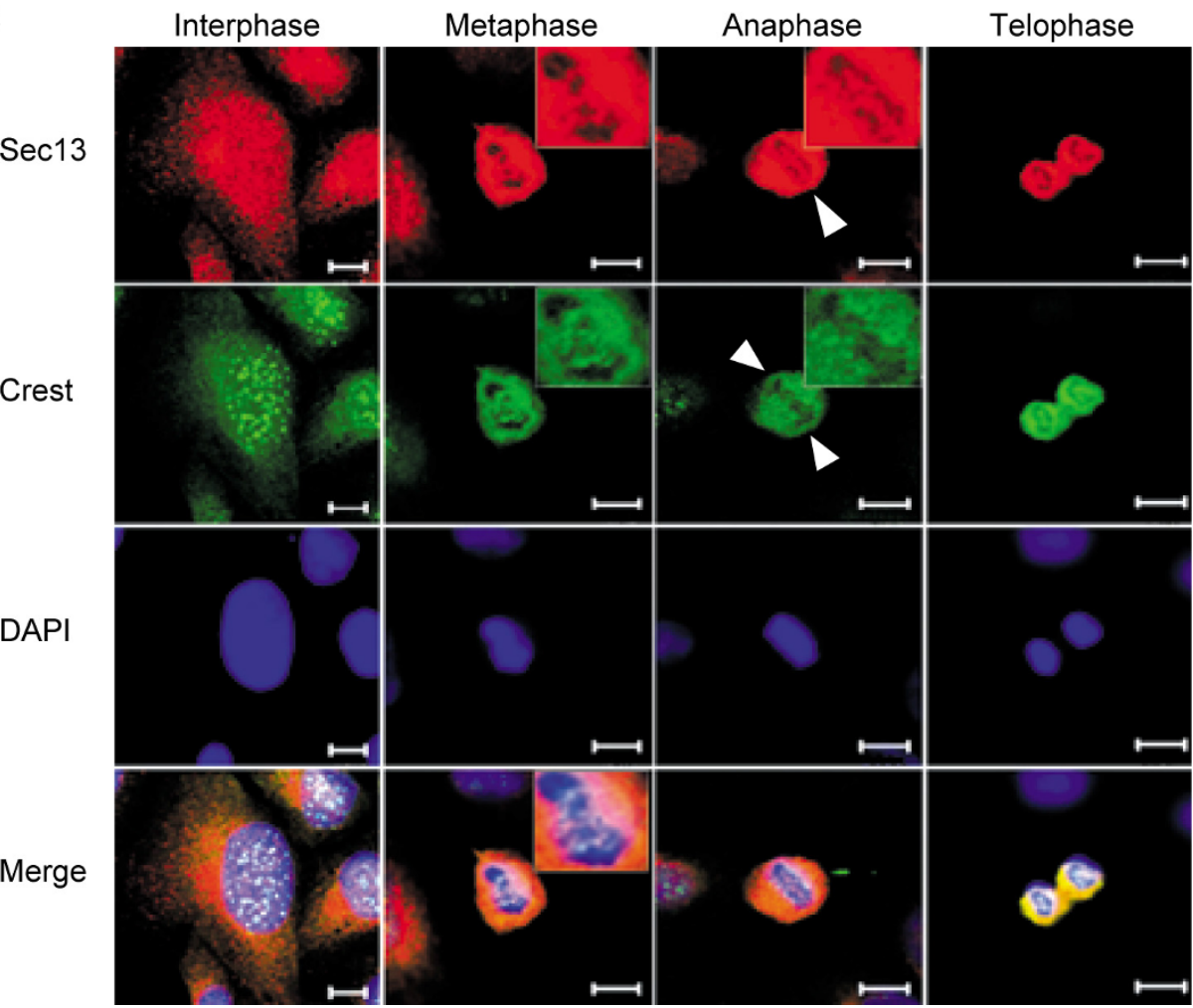

Figure 1. The expression and localization of Sec13 in U2OS cells during the cell cycle. (A) Sec13 expression was examined by immunoblotting. Cells arrested with nocodazole were released in a drug-free medium. U20S cells were harvested at the indicated time points. Total cell lysates of an equivalent amount of total protein were analyzed by SDS-PAGE and immunoblotted using anti-Sec13 antibody. Each cell cycle stage was confirmed by FACS analysis. (B) Sec13 localization was examined by indirect immunofluorescence. Cells on coverslip were fixed, and Sec13 was detected with the antibody and the binding was visualized by fluorescein-isothiocyanate-conjugated secondary antibody. Anti-Crest antibody was used to stain the centromere, and chromosomes were stained with DAPI. Bar $=10 \mathrm{um}$.

$\mathrm{ng} / \mathrm{ml}$ ) for the checkpoint activation. The GFP positive cells were observed at $16 \mathrm{~h}$ after drug treatment to determine the mitotic index. As expected, cells containing the GFP vector alone appeared to be arrested at metaphase by $70 \%$ in the presence of nocodazole (Figure 2A). However, only $30 \%$ of GFP-positive cells had condensed chromosomes under the spindle poison condition, and a significant number of cells expressing the GFP-Sec13 protein showed G1-like morphology (Figure 2A, B), indicating that most of the cells containing the GFP-Sec13 bypassed the obstacle at metaphase and exited mitosis. Dominant negative mutants of the spindle checkpoint components showed a similar pattern in the nocodazole-treated cells (Taylor et al., 1997; Jin et al., 1998). These observations imply that the activity of Sec13 in mitosis overcomes the mitotic inhibition produced by the spindle checkpoint. Thus, Sec13 may serve as an activator for the transition of metaphase to anaphase.

\section{Genomic instability induced by Sec13}

In order to determine genomic instability in cells expressing exogenous Sec13 protein, we conducted 
A

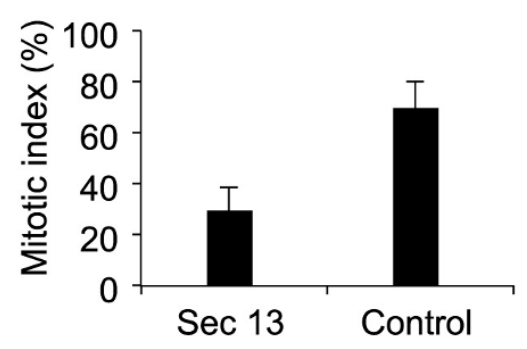

C

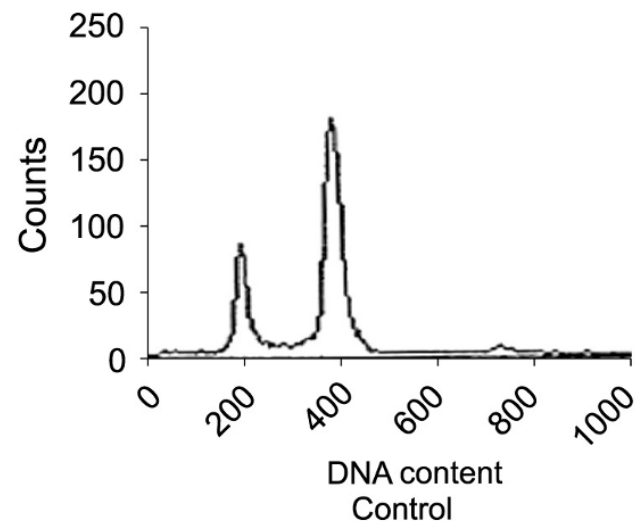

DAPI
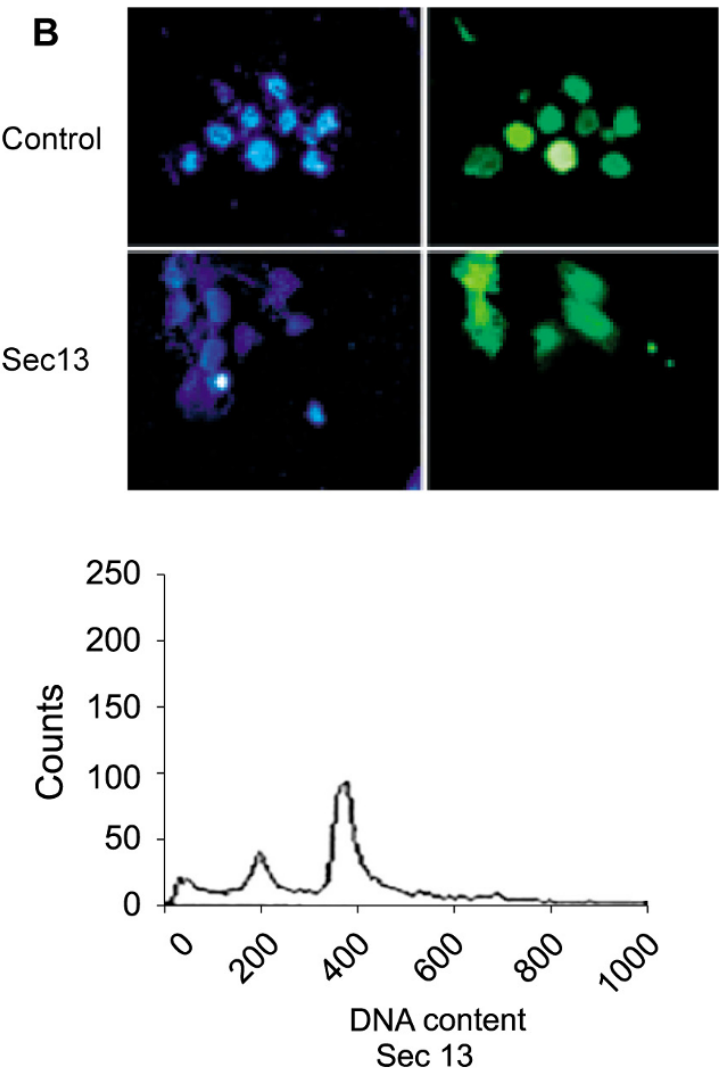

D

\begin{tabular}{|c|c|}
\hline GFP & DAPI \\
\hline -Sec13 & DAbulin \\
\hline Tubrge
\end{tabular}
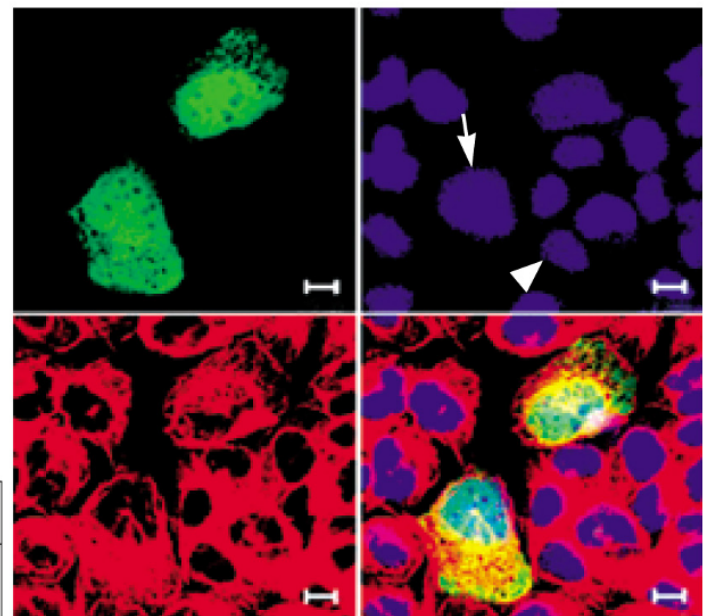

Figure 2. Genomic instability induced by Sec13. (A) Mitotic index was measured by the quantitative analysis of condensed chromosome. U20S cells were transfected with GFP-Sec13 (Sec13) or GFP alone (control). Cells were incubated in the presence of $100 \mathrm{ng} / \mathrm{ml}$ nocodazole for $16 \mathrm{~h}$ and rapidly fixed. The condensed chromosome was scored for 300 cells. Results are the mean \pm SD from three separate experiments. (B) Fluorescence signal was observed in U2OS cells for exogenous GFP-Sec13 (Sec13) or GFP alone (control) in the presence of nocodazole. Corresponding DAPI staining is shown on the left. (C) Cells were transfected with GFP or GFP-Sec13 plasmid for $24 \mathrm{~h}$ and treated with nocodazole for an additional $16 \mathrm{~h}$. After fixation, cells were stained with propidium iodide, and analyzed by flow cytometry. A gate was set in each to select for GFP-positive cells. (D) The morphology of U20S cells expressing GFP-Sec13 in the absence of nocodazole. At $36 \mathrm{~h}$ post-transfection with GFP-Sec13 plasmid, cells were observed by fluorescence microscope. DAPI staining indicates chromosomes. Bar, $10 \mu \mathrm{m}$. Note the morphology of nuclei in U2OS cells expressing the endogenous Sec13 (arrow head) and exogenous Sec13 (arrow). Each of three independent experiments showed identical results. 
FACS analysis in U2OS cells after drug treatment. GFP-positive cells were sorted and their cell cycle profile was analyzed. As expected, the G2/M population in cells expressing Sec13 was dominant in the presence of nocodazole (Figure $2 \mathrm{C}$ ), indicating that these cells entered $\mathrm{G} 1$ with tetraploid status. $\mathrm{Re}$ pressed Mad2 could induce mitotic exit without chromosome segregation producing tetraploid $\mathrm{G} 1$ cells (Gorbsky et al., 1998). In addition, some cells expressing GFP-Sec13 accumulated in the sub-G1 population. This phenotype could be dependent on p53 status in ensuring genomic integrity. The loss of p53 protein could lead to gross aneuploidy (Margolis et al., 2003; Seo and Jung, 2004). U2OS cells used in our study had intact $p 53$ protein so that cells underwent tetraploidy $\mathrm{G} 1$-like phase and induced apoptosis in drug arrested mitotic cells, whereas REF-52 cells in which p53 is inactive do not arrest in G1 following tetraploidization and rapidly progress to aneuploidy in evasion of mitotic arrest by inhibitors of microtubule assembly (Minn et al., 1996; Andreassen et al., 2001; Hong et al., 2004).

Even without ectopic disruption of the spindle checkpoint using microtubule toxin, the endogenous spindle checkpoint is always active in every cell cycle regulating the onset of anaphase. Therefore, in order to investigate whether Sec13 might also be evident during normal mitotic progress, we observed chromosomal morphology in the absence of nocodazole using fluorescence microscope. As shown in Figure $2 \mathrm{D}$, in the GFP-Sec13 expressing cells we observed giant nuclei in the absence of nocodazole compared to cells containing endogenous Sec13. Clearly, further studies are needed to elucidate the specific roles of Sec13 for mitotic progression: i.e. to show interaction with other mitotic proteins. According to our preliminary study, Sec13 interacted with p55CDC protein in yeast two-hybrid system (data not shown). p55CDC/ hCdc20, which is a homolog of CDC20 in Saccharomyces cerevisiae and Schizosaccharomyces pombe SIp1 is an activator of anaphase promoting complex (APC) for ubiquitin ligase activity via degradation of substrate target proteins such as Pds1 (Kramer et al., 1998). Mad2, a key component of the spindle checkpoint, inhibits the APC by titrating its activator, p55CDC (Kim et al., 1998; Luo et al., 2000). Therefore, this observation suggests that there may be an association of p55CDC with Sec13 during mitosis. Interestingly, both proteins have a WD repeat domain known for protein-protein interaction (Saxena et al., 1996). Therefore, it is possible that p55CDC may interact with Sec13 via a WD repeat domain. Further study is required to determine how Sec13 associates and regulates the spindle checkpoint during mitosis. In summary, our current results suggest that the Sec13 effectively blocks the spindle checkpoint response, leading to genomic instability.

\section{Acknowledgement}

We thank Dr. Tang for providing the Sec13 primary antibody. This work was supported by the Korea
Research Foundation Grant (KRF-2002-003-C00104).

\section{References}

Andreassen PR, Lohez OD, Lacroix FB, Margolis RL. Tetraploid state induces p53-dependent arrest of nontransformed mammalian cells in G1. Mol Biol Cell 2001;12: 1315-28

Asada S, Yagura T. Golgi membrane vesicles in HeLa mitotic cells are identified with monoclonal antibody made against Golgi cisternal membrane protein p138. Cell Struct Funct 1995;20:445-53

Belgareh N, Rabut G, Bai SW, van Overbeek M, Beaudouin J, Daigle N, Zatsepina OV, Pasteau F, Labas V, FromontRacine M, Ellenberg J, Doye V. An evolutionarily conserved NPC subcomplex, which redistributes in part to kinetochores in mammalian cells. J Cell Biol 2001;154:1147-60

Boehmer T, Enninga J, Dales S, Blobel G, Zhong H. Depletion of a single nucleoporin, Nup107, prevents the assembly of a subset of nucleoporins into the nuclear pore complex. Proc Natl Acad Sci USA 2003;100:981-5

Campbell MS, Chan G.K, Yen TJ. Mitotic checkpoint proteins HsMAD1 and HsMAD2 are associated with nuclear pore complexes in interphase. J Cell Sci 2001;114:953-63

Enninga J, Levay A, Fontoura BM. Sec13 Shuttles between the Nucleus and the Cytoplasm and Stably Interacts with Nup96 at the Nuclear Pore Complex. Mol Cell Biol 2003; 23:7271-84

Gorbsky GJ, Chen RH, Murray AW. Microinjection of antibody to Mad2 protein into mammalian cells in mitosis induces premature anaphase. J Cell Biol1998;141:1193-205

Hong SE, Hong BS, Kim DC, Rho MS, Park JI, Rha SH, Jun HS, Jeong JS. Involvement of mitogen-activated protein kinases and p21 Waf1 in hydroxyurea-induced G1 arrest and senescence of McA-RH7777 rat hepatoma cell line. Exp Mol Med 2004;36:493-8

louk T, Kerscher O, Scott RJ, Basrai MA, Wozniak RW. The yeast nuclear pore complex functionally interacts with components of the spindle assembly checkpoint. J Cell Biol 2002;159:807-19

Jin DY, Spencer F, Jeang KT. Human T cell leukemia virus type 1 oncoprotein Tax targets the human mitotic checkpoint protein MAD1. Cell; 93:81-91

Kim SH, Lin DP, Matsumoto S, Kitazono A, Matsumoto T. Fission yeast SIp1: an effector of the MAD2-dependent spindle checkpoint. Science 1998;279:1045-7

Kramer ER, Gieffers C, Holzl G, Hengstschlager M, Peters $\mathrm{JM}$, Activation of the human anaphase-promoting complex by proteins of the CDC20/Fizzy family. Curr Biol 1998;8:1207-10

Lederkremer GZ, Cheng Y, Petre BM, Vogan E, Springer $S$, Schekman R, Walz T, Kirchhausen, T. Structure of the Sec23p/24p and Sec13p/31p complexes of COPII. Proc Natl Acad Sci USA 2001;98:10704-9

Li Y, Benezra R. Identification of a human mitotic checkpoint gene: hsMAD2. Science 1996;274:246-8 
Luo X, Fang G., Coldiron M, Lin $Y, Y u H$, Kirschner M W, Wagner $G$. Structure of the Mad2 spindle assembly checkpoint protein and its interaction with $\mathrm{Cdc} 20$. Nat Struct Biol 2000;7:224-9

Margolis RL, Lohez OD, Andreassen PR. G1 tetraploidy checkpoint and the suppression of tumorigenesis. J Cell Biochem 2003;88:673-83

Minn AJ, Boise LH, Thompson CB. Expression of Bcl-x and loss of p53 can cooperate to overcome a cell cycle checkpoint induced by mitotic spindle damage. Genes Dev 1996;10:2621-31

Poloni D, Simanis VA. DMSO-sensitive conditional mutant of the fission yeast orthologue of the Saccharomyces cerevisiae SEC13 gene is defective in septation. FEBS Lett 2002;511: $85-9$

Pryer NK, Salama NR, Schekman R, Kaiser CA, Cytosolic Sec13p complex is required for vesicle formation from the endoplasmic reticulum in vitro. J Cell Biol 1993;120:865-75

Seo YR, Jung HJ. The potential roles of p53 tumor suppressor in nucleotide excision repair (NER) and base excision repair (BER). Exp Mol Med 2004;36:505-9

Siniossoglou S, Wimmer C, Rieger M, Doye V, Tekotte H, Weise C, Emig S, Segref A, Hurt EC. A novel complex of nucleoporins, which includes Sec13p and a Sec13p homolog, is essential for normal nuclear pores. Cell 1996;84:265-75

Swaroop A, Yang-Feng TL, Liu W, Gieser L, Barrow LL,
Chen KC, Agarwal N, Meisler MH, Smith DI. Molecular characterization of a novel human gene, SEC13R, related to the yeast secretory pathway gene SEC13, and mapping to a conserved linkage group on human chromosome 3p24-p25 and mouse chromosome 6. Hum Mol Genet 1994; 3:1281-6

Tang BL, Peter F, Krijnse-Locker J, Low SH, Griffiths G, Hong W. The mammalian homolog of yeast Sec13p is enriched in the intermediate compartment and is essential for protein transport from the endoplasmic reticulum to the Golgi apparatus. Mol Cell Biol 1997;17:256-66

Taylor SS, McKeon F, Kinetochore localization of murine Bub1 is required for normal mitotic timing and checkpoint response to spindle damage. Cell; 89:727-35

Salina D, Enarson P, Rattner JB, Burke B. Nup358 integrates nuclear envelope breakdown with kinetochore assembly. J Cell Biol 2003;162:991-1001

Saxena K, Gaitatzes C, Walsh MT, Eck M, Neer EJ, Smith TF. Analysis of the physical properties and molecular modeling of Sec13: A WD repeat protein involved in vesicular traffic. Biochemistry 1996;35:15215-21

Zaal KJ, Smith CL, Polishchuk RS, Altan N, Cole NB, Ellenberg J, Hirschberg K, Presley JF, Roberts TH, Siggia E, Phair RD, Lippincott-Schwartz J. Golgi membranes are absorbed into and reemerge from the ER during mitosis. Cell 1999:99:589-601 